\title{
Influence of LVAD function on mechanical unloading and electromechanical delay: a simulation study
}

\author{
Aulia Khamas Heikhmakhtiar ${ }^{1}$ - Ah Jin Ryu ${ }^{2}$ Eun Bo Shim ${ }^{2}$ - Kwang-Soup Song ${ }^{1}$. \\ Natalia A. Trayanova ${ }^{3} \cdot$ Ki Moo Lim ${ }^{1}$
}

Received: 29 December 2016 / Accepted: 4 October 2017 / Published online: 3 November 2017

(C) The Author(s) 2017. This article is an open access publication

\begin{abstract}
This study hypothesized that a left ventricular assist device (LVAD) shortens the electromechanical delay (EMD) by mechanical unloading. The goal of this study is to examine, by computational modeling, the influence of LVAD on EMD for four heart failure (HF) cases ranging from mild HF to severe HF. We constructed an integrated model of an LVAD-implanted cardiovascular system, then we altered the $\mathrm{Ca}^{2+}$ transient magnitude, with scaling factors $1,0.9,0.8$, and 0.7 representing HF1, HF2, $\mathrm{HF} 3$, and HF4, respectively, in order of increasing HF severity. The four HF conditions are classified into two groups. Group one is the four HF conditions without LVAD, and group two is the conditions treated with continuous LVAD pump. The single-cell mechanical responses showed that EMD was prolonged with the higher load. The findings indicated that in group one, the HF-induced $\mathrm{Ca} 2+$ transient remodeling prolonged the mechanical activation
\end{abstract}

A. K. Heikhmakhtiar and A. J. Ryu contributed equally to this paper (co-first author).

Electronic supplementary material The online version of this article (https://doi.org/10.1007/s11517-017-1730-y) contains supplementary material, which is available to authorized users.

Ki Moo Lim

kmlim@kumoh.ac.kr

1 Department of IT Convergence Engineering, Kumoh National Institute of Technology, Yangho-dong, Gumi, Gyeongbuk 730-701, Republic of Korea

2 Department of Mechanical \& Biomedical Engineering, Kangwon National University, Cuncheon, Republic of Korea

3 Department of Biomedical Engineering, Johns Hopkins University, Baltimore, MD, USA time (MAT) and decreased the contractile tension, which reduced the left ventricle (LV) pressure, and increased the end-diastolic strain. In group two, LVAD shortened MAT of the ventricles. Furthermore, LVAD reduced the contractile tension, and end-diastolic strain, but increased the aortic pressure. The computational study demonstrated that LVAD shortens EMD by mechanical unloading of the ventricle.

Keywords Ventricular electromechanical model · Heart failure Calcium transient $\cdot$ Left ventricular assist device

\section{Introduction}

Heart failure (HF) is a chronic and progressive condition, with the heart muscle being unable to pump the appropriate amount of blood to fulfill the needs of the human body [1]. A report from the American Heart Association Statistics Committee and Stroke Statistics Subcommittee concluded that $\mathrm{HF}$ is a major cause of morbidity and mortality, and that it contributes significantly to health expenses around the world [16].

A subset of HF includes dyssynchrony between cardiac depolarization and myofiber shortening, which in turn further increases the severity of HF. The time interval between the local myocyte depolarization (electrical activation) and onset of myofiber shortening (mechanical activation) is termed electromechanical delay (EMD) [8]. Normal EMD is typically about $10 \mathrm{~ms}$, and long EMD implies lack of synchrony in cardiac electromechanical activation and a decrease in ventricular pumping efficacy [8].

Constantino et al. [5] identified four major aspects that contribute to prolonged EMD under dyssynchronous HF conditions: remodeled cardiac structure (both heart shape and fiber structure), altered electrical conduction, deranged 
$\mathrm{Ca}^{2+}$ handling, and increased stiffness of the tissue. The timely application of electrical stimulation (termed as cardiac resynchronization therapy (CRT)) can alter the electrical conduction pattern in the ventricles, provide synchrony, and improve the pumping of the heart. The study by Constantino et al. demonstrated that CRT reduced cardiac EMD by reducing the overall electrical activation time [6]. Furthermore, it also found that deranged $\mathrm{Ca}^{2+}$ handling resulting in a diminished magnitude of the $\mathrm{Ca}^{2+}$ transient, was the primary factor responsible for prolonged EMD. The other three factors had a much smaller contribution to EMD.

An experimental study conducted by Russell et al. in canine and human hearts showed that a mechanical load prolonged the EMD [19]. Although the findings of these studies suggested that EMD decreases if the mechanical load of the ventricles decreases, no research to date has validated this suggestion. A left ventricular assist device (LVAD), used to support cardiac function and improve cardiac output [23], also reduces the mechanical load of the ventricles by enabling an improved pump function. In a previous study of ours, we developed a computational model of the ventricles with LVAD support and showed that the LVAD decreased ventricular after load and improved coronary perfusion [15].

The goal of the present study was to examine, using similar computational modeling, the effect of mechanical load on a single cell and the effect of LVAD on the three-dimensional (3D) distribution of EMD in the four failing heart conditions ranging from mild to severe contractility, and to test the hypothesis that LVAD overall shortens EMD by reducing mechanical afterload. The use of computational modeling overcomes the inability of experimental methodologies to measure and quantify the EMD distribution in the heart.

\section{Methods}

\subsection{Model description}

In this study, the 3D image-based electromechanical model of failing ventricles $[9,22]$ was combined with a lumped model of the circulatory system and LVAD function [15] to construct an integrated model of an LVAD-implanted cardiovascular system. A schematic diagram of the integrated model is shown in Fig. 1. The various components of the combined model are described below. The mathematical equations for the electromechanical model as well as the circulatory system can be found in Suplementary Material.

Electrical model The electromechanical model of the failing ventricles used in this study had two dynamic components, namely electrical components and mechanical components, as described in a previous study [9]. The electrical component of the model simulated the propagation of a transmembrane potential wave by solving the monodomain equations on a finite-element tetrahedral mesh comprising 241,725 nodes and 1,298,751 elements. The monodomain partial differential equation (PDE) describes the current flow through ventricles composed of cells connected via conductive gap junctions. The 2D Purkinje network model proposed by Berenfeld and Jalife1 was then mapped onto a 3D endocardial surface of the ventricles [4]. Electrical wave propagation through the Purkinje fiber was implemented by solving the one-dimensional wave propagation equation that triggered the ventricular activation. The current flow in the ventricular tissue was driven by ion exchange across the cellular membranes. These processes were represented using a human ionic model by Ten Tusscher et al. [21], which described the current flow through ion channels, pumps, and exchangers in the myocyte membrane as well as the subcellular Ca cycling as a set of ordinary differential equations (ODEs). Electrical wave propagation in the heart was determined by simultaneously solving the PDE and set of ODEs in the electrical ventricular mesh. The local $\mathrm{Ca}^{2+}$ transient at each location in the ventricles served as local trigger for the cardiac mechanics model.

Mechanical model The mechanical component of the model simulated ventricular contraction. Ventricular contraction is a result of an active tension generated by the myofilaments of ventricular cells. Ventricular deformation is represented by equations of passive cardiac mechanics, given that the myocardium is an orthotropic (due to the fiber and laminar sheet organization), hyperelastic, and nearly incompressible material with passive mechanical properties defined by an exponential strain-energy function [10]. The model comprised 356 nodes and 172 Hermite elements. The simulation of ventricular contraction was performed by simultaneously solving the active myofilament model equations along with the equations representing passive cardiac mechanics on the finite-element mechanical mesh. The electromechanical model incorporated the biophysical representation of cardiac myofilament dynamics by Rice et al. [18], which represents the excitation-contraction coupling mechanisms (cross-bridge cycling induced by $\mathrm{Ca}^{2+}$ release). A set of ODEs and algebraic equations described the $\mathrm{Ca}^{2+}$ binding to troponin $\mathrm{C}$, cooperation between regulatory proteins, and cross-bridge cycling. In order to take the HF properties into account, we used a dilated failing ventricle based on a magnetic resonance (MR) image $[9,22]$ with the size of a kid's heart, and we scaled the passive scaling constant of the strain-energy function by fivefold to increase the stiffness of the failing myocardium [24]. A lumpedparameter model of the systemic and pulmonic circulatory systems imposed conditions on ventricular volumes and pressure; it was based on a model by Kerckhoffs et al. 


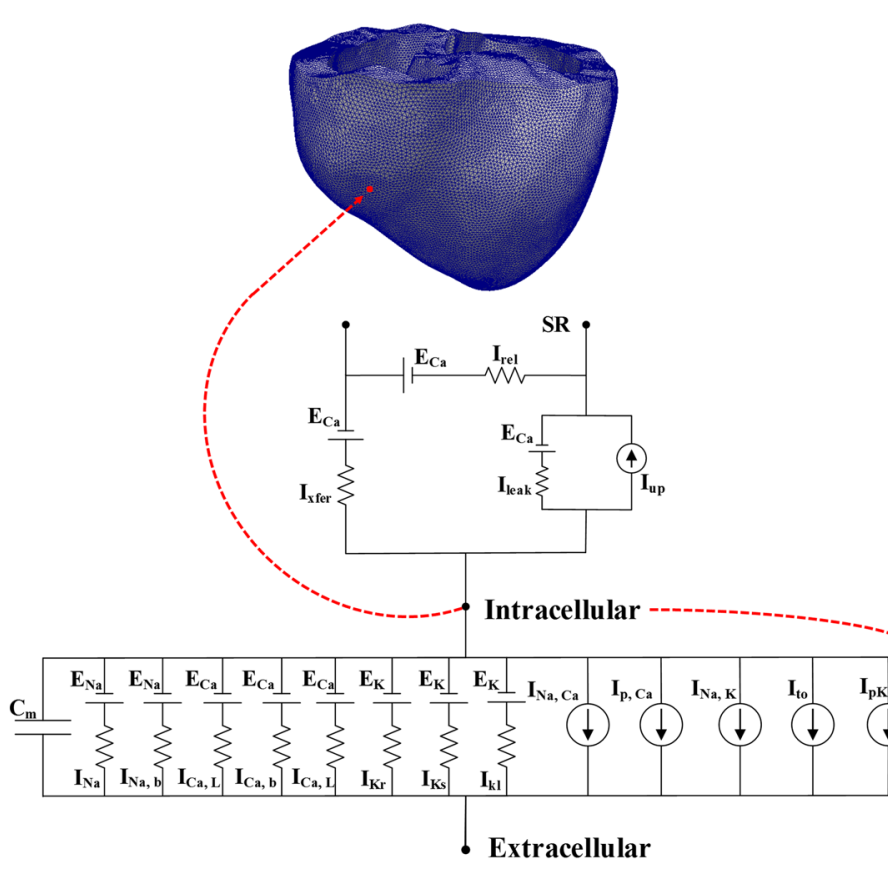

Fig. 1 Schematics of the electrical and mechanical elements coupled with calcium transient. Electrical Element. It represents the currents, pumps, and exchanger of Ten Tusscher ionic model. Fast inward $\mathrm{Na}^{+}$current $\left(\mathrm{I}_{N a}\right)$, background $\mathrm{Na}^{+}$current $\left(\mathrm{I}_{N a, b}\right)$, L-type inward $\mathrm{Ca}^{2+}$ current $\left(\mathrm{I}_{C a, L}\right)$, background $\mathrm{Ca}^{2+}$ current $\left(\mathrm{I}_{C a, b}\right)$, rapid delayed rectifier $\mathrm{K}^{+}$current $\left(\mathrm{I}_{K r}\right)$, slow delayed rectifier $\mathrm{K}^{+}$current $\left(\mathrm{I}_{K s}\right)$, inward rectifier $\mathrm{K}_{1}$ current $\left(\mathrm{I}_{K 1}\right), \mathrm{Na}^{+}-\mathrm{Ca}^{2+}$ exchange current $\left(\mathrm{I}_{N a, C a}\right)$, sarcoplasmic $\mathrm{Ca}^{2+}$ pump current $\left(\mathrm{I}_{p, C a}\right), \mathrm{Na}^{+}$$\mathrm{K}^{+}$exchange current $\left(\mathrm{I}_{N a, K}\right)$, transient outward $\mathrm{K}^{+}$current $\left(\mathrm{I}_{t o}\right)$, $\mathrm{K}^{+}$pump current $\left(\mathrm{I}_{p, K}\right), \mathrm{Ca}^{2+}$ release current from the JSR $\left(\mathrm{I}_{r e l}\right)$, $\mathrm{Ca}^{2+}$ leak current from the JSR $\left(\mathrm{I}_{r e l}\right)$, and $\mathrm{Ca}^{2+}$ uptake current into

[13]. We estimated the lumped model with high resistance and with $10 \%$ decrease in compliance to support the HF environment.

LVAD function This electromechanical model was combined with a lumped model of the circulatory system and an LVAD function based on the study Lim et al. [15] to construct an integrated model of the LVAD-implanted cardiovascular system. The LVAD component was connected to the electromechanical and circulatory models through an inlet in LV, and the outlet was attached to the aorta in the circulatory model. Briefly, the LVAD component was modeled as a flow generator. Constant-flow conditions were used to simulate a continuous LVAD. In this study, the failing ventricle geometry from the MRI has the size of a kid's heart. Therefore, we adjusted the LVAD flow rate as $50 \mathrm{~mL} / \mathrm{s}$ as LVAD fully assisted the HF3 and HF4 conditions with this flow rate.

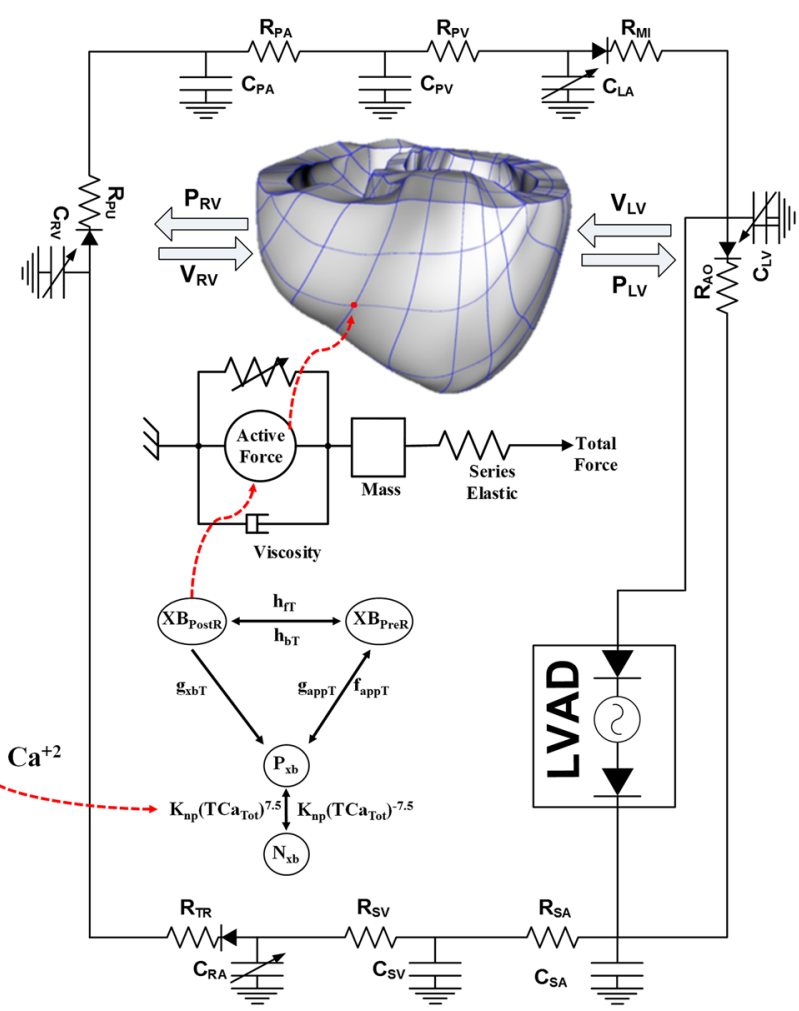

the NSR $\left(\mathrm{I}_{u p}\right)$. Mechanical Element. A schematic diagram of the finite-element ventricular mechanical model coupled with the circulatory and LVAD models. PRV RV pressure, VRV RV volume, PLV LV pressure, VLV LV volume, RPA pulmonary artery resistance, CPA pulmonary artery compliance, RPV pulmonary vein resistance, CPV pulmonary vein compliance, RMI mitral valve resistance, CLA left atrium compliance, RAO aortic valve resistance, RSA systemic artery resistance, CSA systemic artery compliance, RSV systemic vein resistance, CSV systemic vein compliance, RTR tricuspid valve resistance, CRA right atrium compliance, and RPU pulmonary valve resistance

\subsection{Simulation procedure}

Single-cell simulation First, we created four different single-cell myofilament models with different $\mathrm{Ca}^{2+}$ transient magnitudes to explore the effect of the altered $\mathrm{Ca}^{2+}$ transient, found previously to be the main reason for prolonged EMD under HF conditions (see Section 1), on myocardial force generation, shortening, and ATP consumption. The different $\mathrm{Ca}^{2+}$ transient magnitudes represented different levels of severity of HF conditions. The $\mathrm{Ca}^{2+}$ transient magnitude was controlled by a scaling factor, with scaling factors $1,0.9,0.8$, and 0.7 representing HF1, HF2, HF3, and HF4, respectively, in order of increasing HF severity. For each case, the isometric and isotonic contractions of a single-myocardial cell were simulated. To simulate the isometric condition, we set an infinite amount of load to the myofilament model so that the myofilament could not contract. To simulate the isotonic contraction, we set two different load conditions, 
namely, 30 and $50 \mathrm{mN} / \mathrm{mm}$. The computation was performed for 20 cycles for both isometric and isotonic conditions with a basic cycle length of $600 \mathrm{~ms}$. Comparisons between the isometric and isotonic of $30 \mathrm{mN} / \mathrm{mm}$, and isometric and isotonic of $50 \mathrm{mN} / \mathrm{mm}$ were performed at the 20th cycle when the cell had reached a steady-state condition.

3D electromechanics simulation First, we simulated the electrical component by inducing an electrical signal to a ventricular tissue through the Purkinje networks with $600 \mathrm{~ms}$ for one cycle until steady state. The distribution of the Purkinje networks to the ventricle is based on the experimental study by Durrer et al. [7]. Here, we altered the electrical conduction by adjusting the conduction velocity in the ventricle tissue as $60 \mathrm{~cm} / \mathrm{s}$ (normally $70 \mathrm{~cm} / \mathrm{s}[12$, $20,21])$. The $3 \mathrm{D}$ ventricular modeling involved the generation of two groups of models, namely, a control group, which consists of the four HF conditions with no mechanical support and an LVAD group (the four HF conditions treated with LVAD). In a manner similar to the singlemyocardial cell simulations, four different $\mathrm{Ca}^{2+}$ transient magnitudes were used to simulate mild to severe HF (HF1 to HF4). Then we took the electrical activation time from each node, and set the $\mathrm{Ca}^{2+}$ transient information as input to the mechanical simulation. We simulated the mechanical contraction model for $42 \mathrm{~s}$ to obtain a steady-state condition with the same cycle length (600 ms). A continuous LVAD with a pumping flow rate of $3 \mathrm{~L} / \mathrm{min}$ was assumed. Thus, $3 \mathrm{D}$ ventricular simulations were executed for eight different conditions, and model responses including spatial distribution of the myocardial tension and contractile adenosine triphosphate (ATP) consumption, strain distribution, and hemodynamic responses (blood pressure, volume, and flow rate) were compared during the last cycle under steady-state condition. The ATP consumption rates were calculated as the product of the cross-bridge detachment rate and singleoverlap fraction of thick filaments from each node during the end of systolic volume (ESV) based on the method by Rice et al. [18].

Figure 2a shows the electrical activation time (EAT), mechanical activation time (MAT) and EMD. As in Gurev et al. [8], the as EMD was defined as the time interval between the local myocyte depolarization or EAT and the local myofiber shortening or MAT. The local EAT was defined as the transmembrane voltage exceeding $0 \mathrm{mV}$, and the onset of myofiber shortening was defined as the time instant at which the myofiber was shortened to $10 \%$ of its maximal value. The EMD was obtained by subtracting EAT from MAT at every point in the ventricles. Finally, the 3D distributions of EAT, MAT, and EMD were compared between the control group and the LVAD for the four different levels of HF severity.
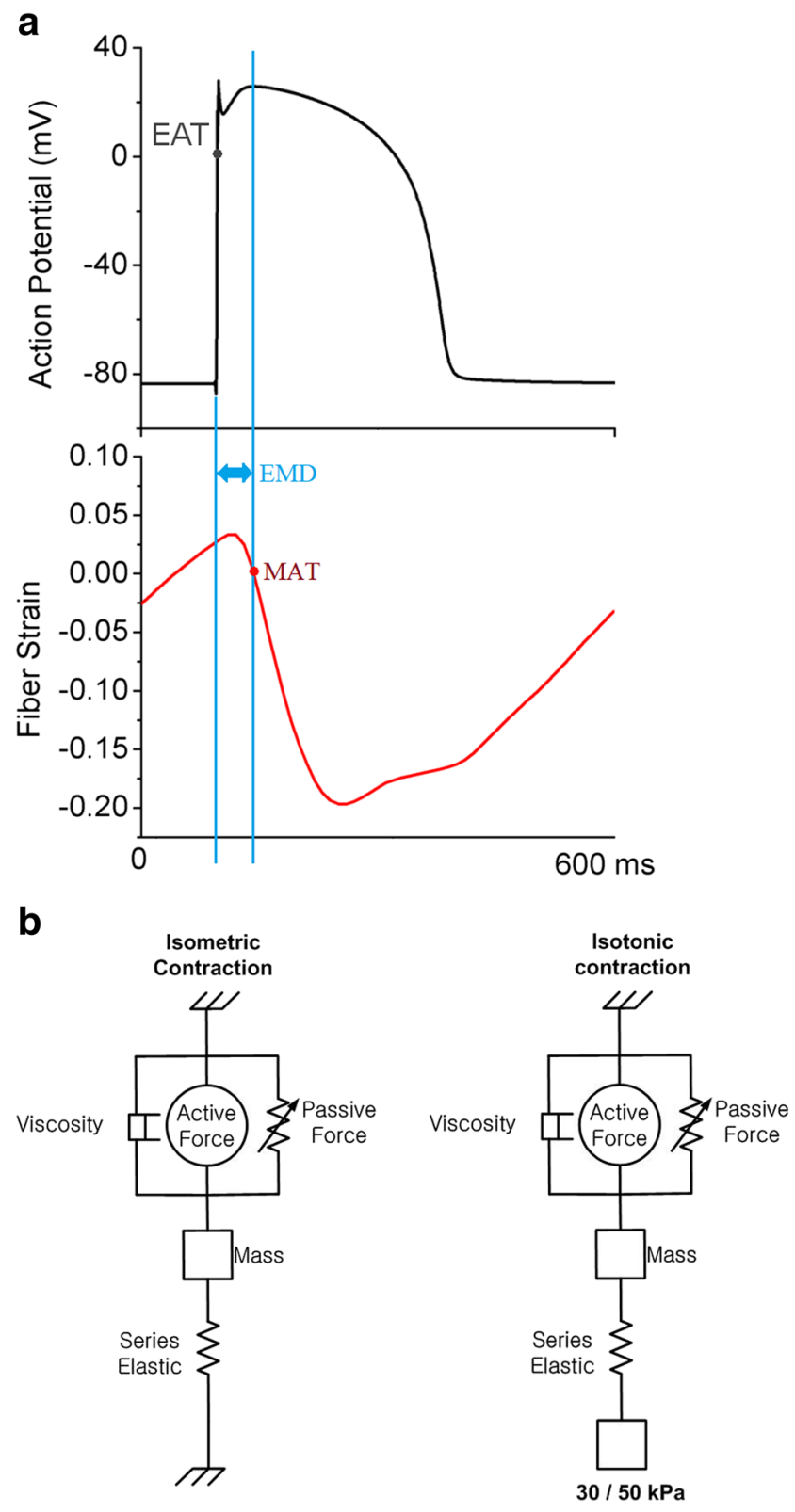

Fig. 2 a Electrical activation time (EAT) is the time of cardio myocell depolarization (with threshold above $0 \mathrm{mV}$ ), mechanical activation time (MAT) is defined as the myofiber shortening at $10 \%$, and electromechanical delay (EMD) is the interval between MAT and EAT. b Isometric contraction (left side) is expressed as an active force although the cell does not contract due to the load. Isotonic contraction (right side) is expressed as an active force from the cross bridge, with a specific load (30 or $50 \mathrm{kPa}$ ) and forming a contraction

\section{Results}

Single-cell responses Figures 3 and 4 show two cycles of $\mathrm{Ca}^{2+}$ transient, myocardial tension, equivalent cell length, and contractile ATP consumption rate for all levels of HF severity (from HF1 to HF4) under 30 and $50 \mathrm{mN} / \mathrm{mm}$ load conditions, respectively. The first cycle is a steady-state 
Fig. 3 Two cycles of $\mathrm{Ca}^{2+}$ transient, myocardial tension (first for isometric contraction and second for isotonic contraction with $30 \mathrm{kPa}$ load), equivalent cell length, and contractile ATP consumption rate for different levels of $\mathrm{HF}$ severity (from HF1 to HF4)
Fig. 4 Two cycles of $\mathrm{Ca}^{2+}$ transient, myocardial tension (first for isometric contraction and second for isotonic contraction with $50 \mathrm{kPa}$ load), equivalent sarcomere length, and contractile ATP consumption rate for different levels of $\mathrm{HF}$ severity (from HF1 to HF4)

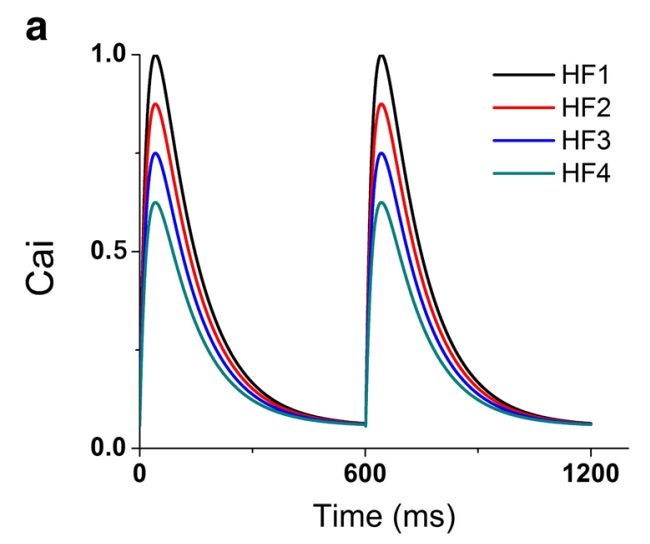

b
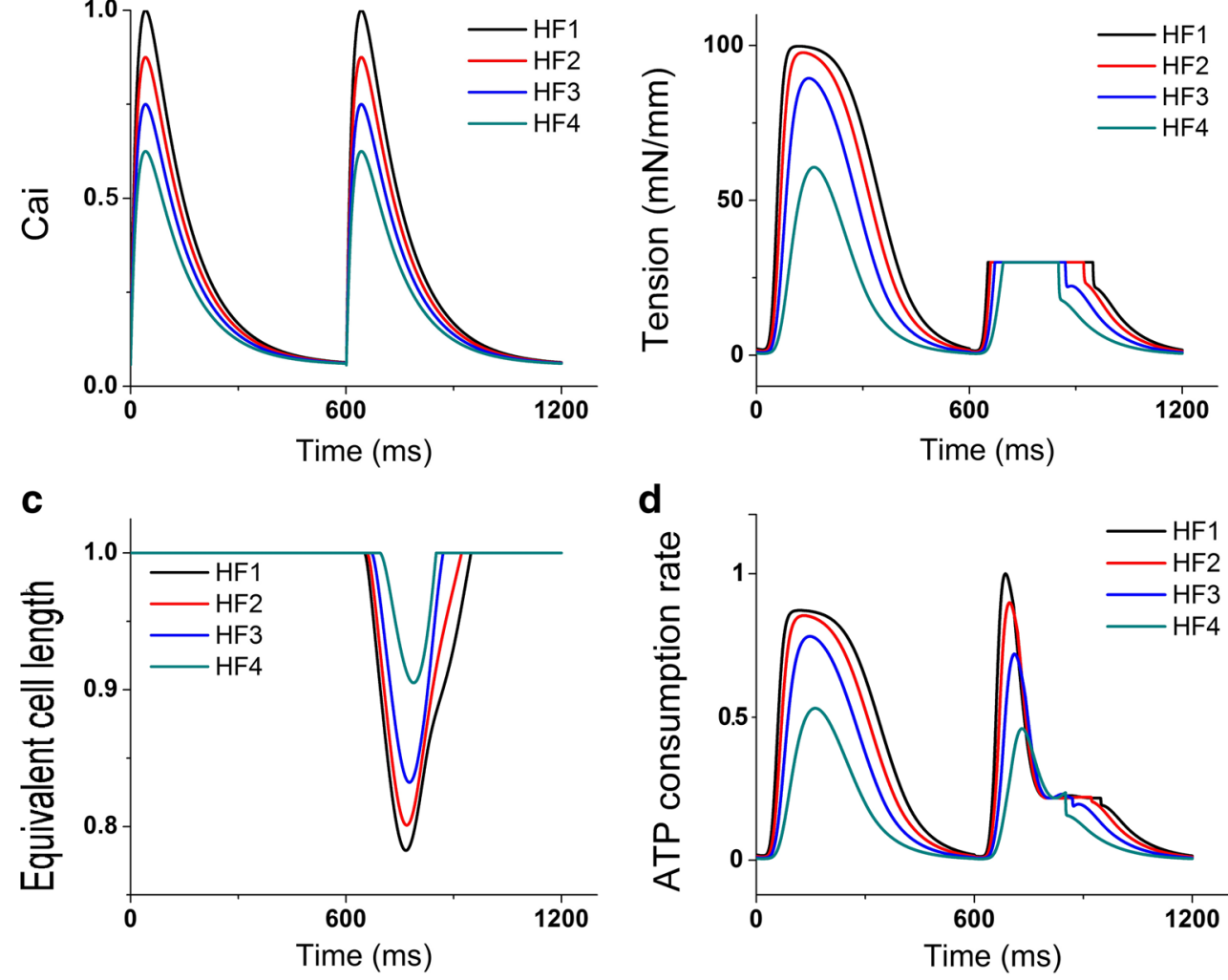

d

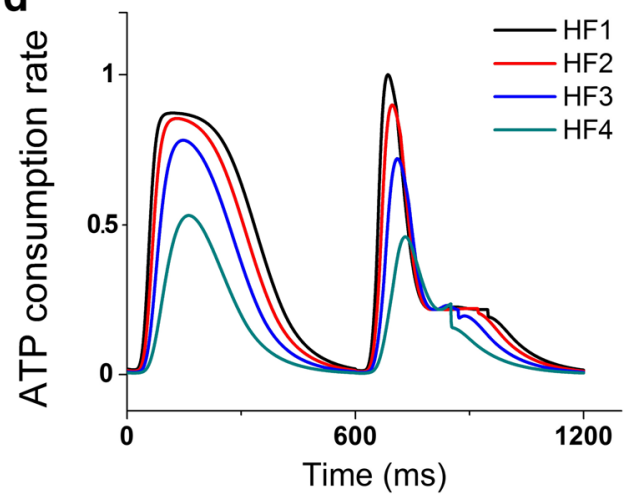

b
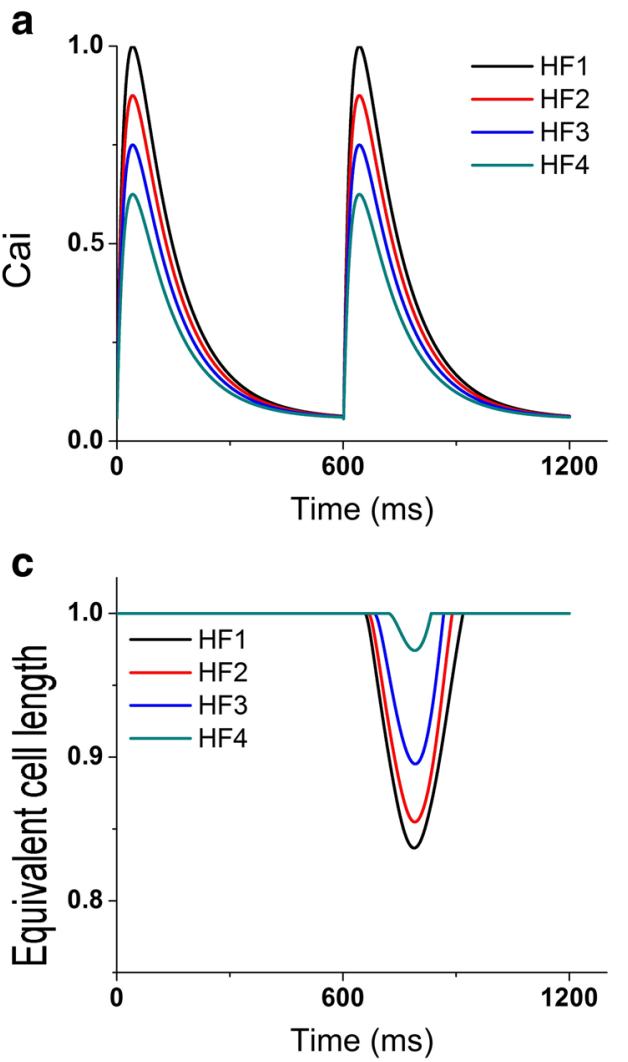

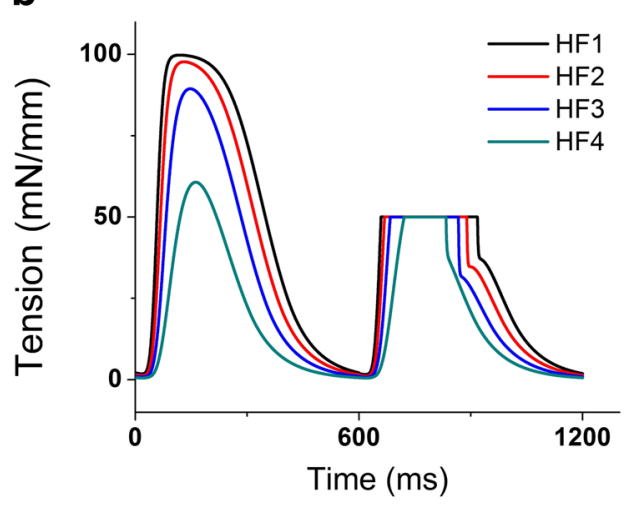

d

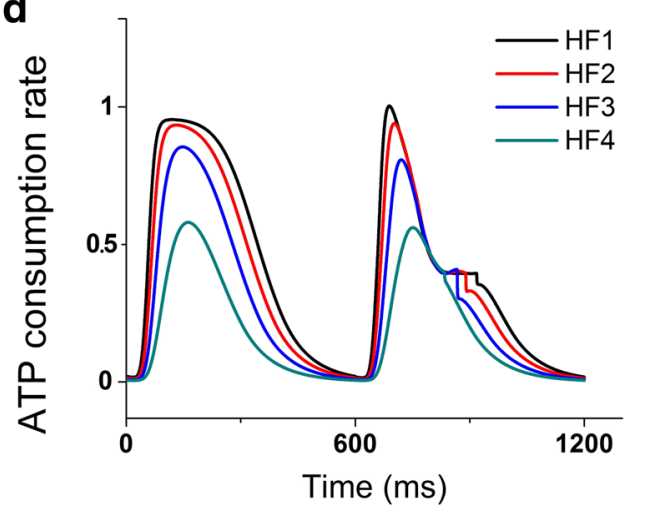


Table 1 Single-cell peak time value of $\mathrm{Ca}$, tension, equivalent cell length, and ATP with $30 \mathrm{kPa}$ load (ms)

\begin{tabular}{|c|c|c|c|c|c|c|c|c|}
\hline \multirow[t]{2}{*}{ HF level } & \multicolumn{2}{|l|}{$\mathrm{Ca}^{2+}$} & \multicolumn{2}{|l|}{ Tension } & \multicolumn{2}{|c|}{ Equivalent cell length } & \multicolumn{2}{|l|}{ ATP } \\
\hline & Isometric & Isotonic & Isometric & Isotonic & Isometric & Isotonic & Isometric & Isotonic \\
\hline HF1 & 41 & 41 & 115 & 52 & 0 & 166 & 114 & 85 \\
\hline HF2 & 41 & 41 & 130 & 60 & 0 & 167 & 127 & 96 \\
\hline HF3 & 41 & 41 & 146 & 72 & 0 & 175 & 147 & 110 \\
\hline HF4 & 41 & 41 & 161 & 95 & 0 & 188 & 161 & 129 \\
\hline
\end{tabular}

condition of isometric contraction while the second cycle is the steady state of isotonic contraction. As shown in the figures, HF1 was associated with the highest myocardial tension (Figs. $3 \mathrm{~b}$ and $4 \mathrm{~b}$ ). It also had the highest ATP consumption rate (Figs. $3 \mathrm{~d}$ and $4 \mathrm{~d}$ ), and hence, the sarcomere shortened the least amount under the isotonic contraction, compared to that of other HF severity levels (Figs. 3c and $4 \mathrm{c}$ ). Overall, a greater $\mathrm{Ca}^{2+}$ transient magnitude resulted in a higher myocardial tension, shortening amount, and contractile ATP consumption rate. In the isotonic contraction with $30 \mathrm{mN} / \mathrm{mm}$ load condition, the shortening values of the sarcomere under the HF2, HF3, and HF4 conditions were 9, 33, and 56\% lower compared to that under the HF1 condition, respectively. In the isotonic contraction with the load of $50 \mathrm{mN} / \mathrm{mm}$, the shortening values of the sarcomere under the HF2, HF3, and HF4 conditions were 11,36 , and $84 \%$ lower compared to that under the HF1 condition, respectively. Furthermore, the shortening values of the sarcomere with $50 \mathrm{mN} / \mathrm{mm}$ load condition were 75 , 73,62 , and $27 \%$ lower compared to the isotonic contraction with $30 \mathrm{mN} / \mathrm{mm}$ load condition for the HF1, HF2, HF3, and HF4 conditions, respectively.

The $\mathrm{Ca}^{2+}$ transient magnitude affected not only the tension amplitude, the length shortening, and the ATP consumption rate, but also the time to reach peak values. Tables 1 and 2 show times to reach the peak value of $\mathrm{Ca}^{2+}$ transient, myocardial tension, equivalent sarcomere length, and contractile ATP consumption rate for the loads of 30 and $50 \mathrm{mN} / \mathrm{mm}$, respectively. Larger $\mathrm{Ca}^{2+}$ transient resulted in faster times-to-peak of myocardial tension, length shortening, and ATP consumption rate. The results were consistent with those obtained by a previous study by Russel et al. [19], which showed that mechanical load contributed to prolonged EMD. We also compared the sarcomere shortening time-to-peak during isotonic contraction with loads of 30 and $50 \mathrm{mN} / \mathrm{mm}$. In HF1 and HF2, the cell with a load of $30 \mathrm{mN} / \mathrm{mm}$ shortened 11.7 and $11.6 \%$ faster, respectively, than a cell with load $50 \mathrm{mN} / \mathrm{mm}$. In HF3, the cell with a load of $30 \mathrm{mN} / \mathrm{mm}$ shortened $7.4 \%$ faster when compared with the cell with a load of $50 \mathrm{mN} / \mathrm{mm}$. However, in HF4, the sarcomeres of both cells shortened the same amount.

3D ventricular responses Figure 5 shows the calculated transmural distribution of myocardial tension (Fig. 5a), contractile ATP consumption rate (Fig. 5b), and strain (Fig. 5c), as well as the pressure waveforms of LV and aorta as a function of time (Fig. 5d is for the control group and Fig. 5e for the LVAD therapy group), and the overall ATP consumption rate of all nodes in one cycle (Fig. 5f), LV stroke work (Fig. 5g), and LV pressure-volume loop (Fig. 5h refers to the control group and Fig. 5i to the LVAD therapy group) for all HF severities. The myocardial tension and contractile ATP consumption rate distribution were obtained at end-systole, while the strain distribution was obtained at end-diastole.

The results indicated that tension and ATP consumption rate were decreased in the LVAD and control groups following the decrease in $\mathrm{Ca}^{2+}$ transient magnitude. For the same HF severity condition, LVAD decreased tension and ATP consumption (Fig. 5a and b). However, strain increased when $\mathrm{Ca}^{2+}$ transient was decreased. The LVAD treatment reduced ventricular strain for severe HF (Fig. 5c).

Figure 5d, e shows the steady-state responses of the LV pressure (black lines) and aortic pressure (red lines) in the control and LVAD groups, respectively. As illustrated in Fig. 5d, under the HF1 condition, the LV peak pressure was $145 \mathrm{mmHg}$, and the aortic pressure was between 109 and $140 \mathrm{mmHg}$. Under the HF2 condition, these numbers were $124 \mathrm{mmHg}$, and between 94 and $120 \mathrm{mmHg}$, respectively.
Table 2 Single-cell peak time value of $\mathrm{Ca}$, tension, equivalent cell length, and ATP with $50 \mathrm{kPa}$ load (ms)

\begin{tabular}{|c|c|c|c|c|c|c|c|c|}
\hline \multirow[t]{2}{*}{ HF level } & \multicolumn{2}{|l|}{$\mathrm{Ca}^{2+}$} & \multicolumn{2}{|l|}{ Tension } & \multicolumn{2}{|c|}{ Equivalent cell length } & \multicolumn{2}{|l|}{ ATP } \\
\hline & Isometric & Isotonic & Isometric & Isotonic & Isometric & Isotonic & Isometric & Isotonic \\
\hline HF1 & 41 & 41 & 115 & 59 & 0 & 188 & 114 & 88 \\
\hline $\mathrm{HF} 2$ & 41 & 41 & 130 & 68 & 0 & 189 & 127 & 101 \\
\hline HF3 & 41 & 41 & 146 & 84 & 0 & 189 & 147 & 119 \\
\hline HF4 & 41 & 41 & 161 & 121 & 0 & 188 & 161 & 149 \\
\hline
\end{tabular}




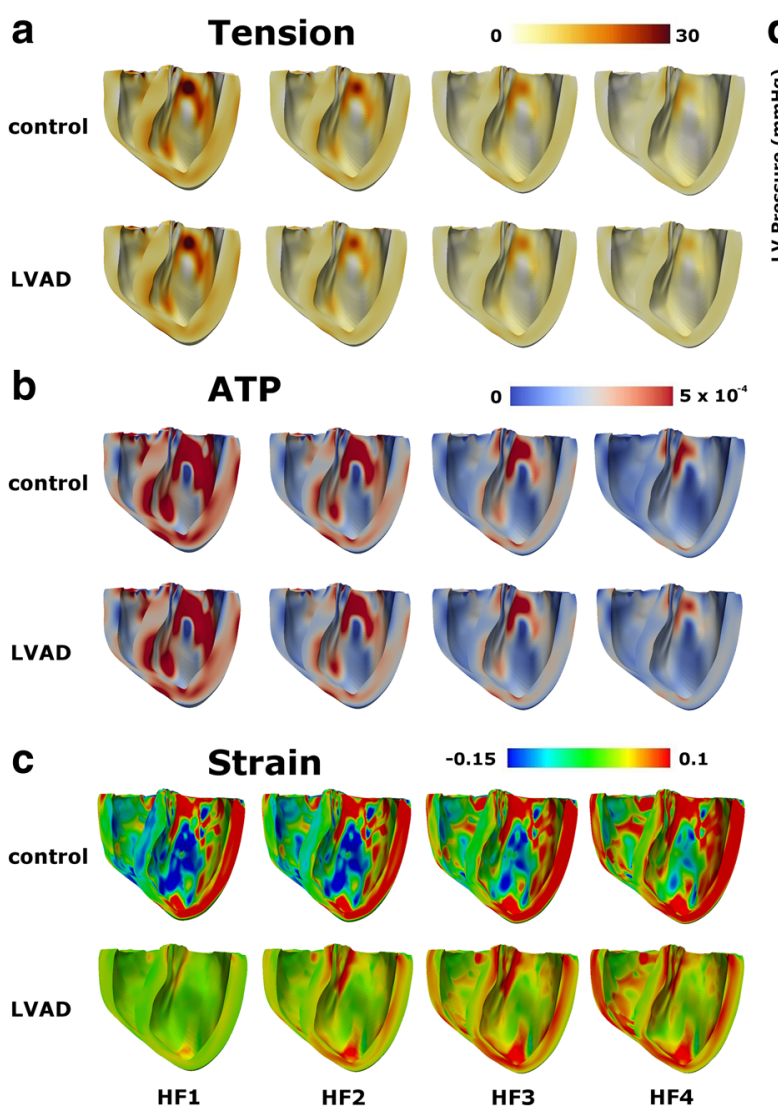

Fig. 5 a Tension at end of systole, b ATP at end of systole, $\mathbf{c}$ strain at the end of diastole, $\mathbf{d}$ LV pressure (black lines) and aortic pressure (red lines) of the control group, e LV pressure (black lines) and aortic
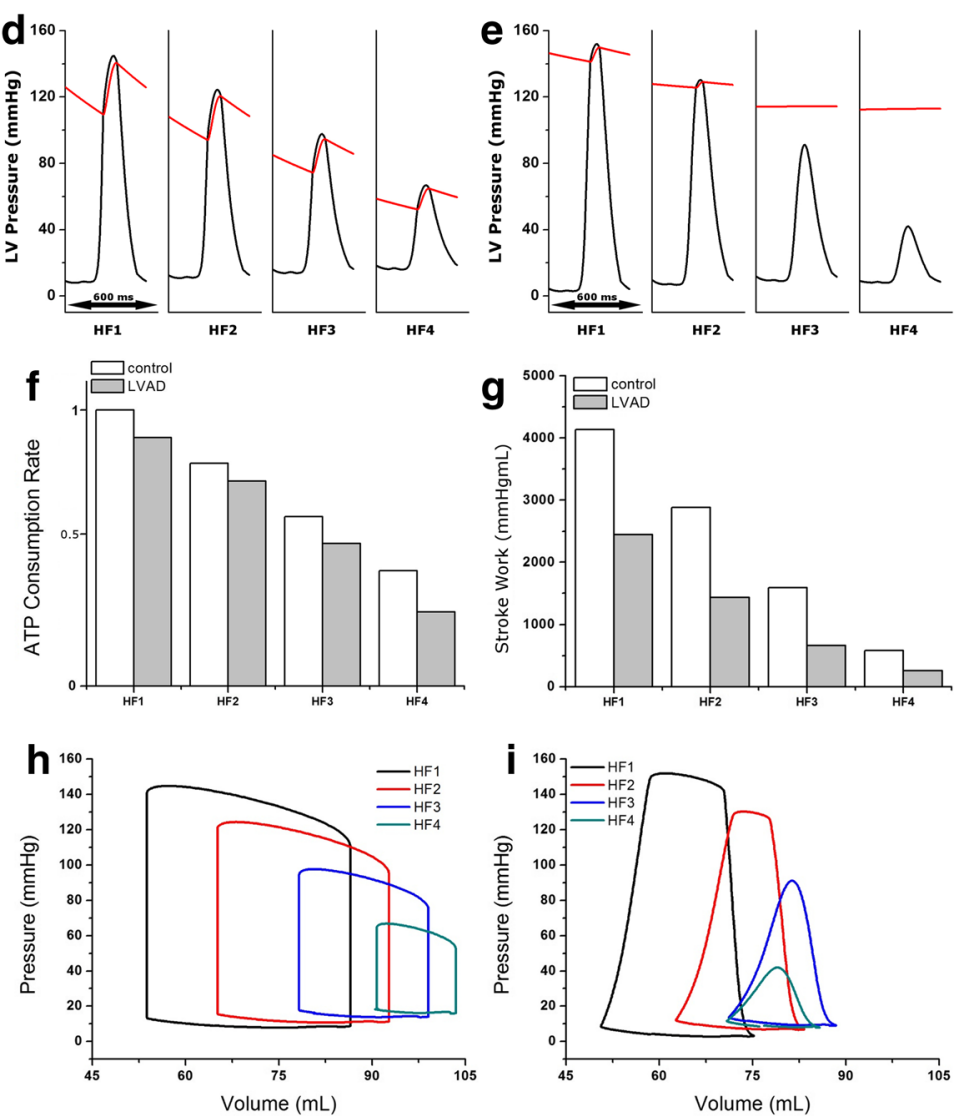

pressure (red lines) in the LVAD group, $\mathbf{f}$ energy consumption in form of the ATP taken from one cycle, $\mathbf{g}$ stroke work, $\mathbf{h}$ pressure-volume loop of control group, and $\mathbf{i}$ pressure-volume loop in the LVAD group
In the HF3 condition, the LV peak pressure was $98 \mathrm{mmHg}$, and the aortic pressure ranged between 74 and $95 \mathrm{mmHg}$. The HF4 condition resulted in the lowest LV and aortic peak pressures, with the LV peak pressure reaching $67 \mathrm{mmHg}$, and the aortic pressure being between 65 and $52 \mathrm{mmHg}$. Additionally, during the diastolic phase, the LV pressure progressively increased from HF1 to HF4.

As Fig. 5e indicates, LVAD fully assisted the LV in pumping blood in the HF3 and HF4 conditions, but for the HF1 and HF2 conditions, it provided only partial assistance. In the HF1 and HF2 conditions, the LV peak pressures were 152 and $130 \mathrm{mmHg}$, respectively. The aortic pressures ranged between 141 and $152 \mathrm{mmHg}$ for the HF1 condition, and between 126 and $129 \mathrm{mmHg}$ for the HF2 condition. In the HF3 and HF4 conditions, the LV peak pressures were 91 and $42 \mathrm{mmHg}$, respectively. However, the aortic pressures remain the same for both the HF3 and HF4 conditions at $113 \mathrm{mmHg}$.

The reason why the LVAD did not fully assist in the distribution of blood under conditions HF1 and HF2 was because the LV pressures in these cases exceeded the aortic pressures during the systolic phase. Under HF3 and
HF4 conditions, the blood volume from the LV was fully unloaded by the LVAD and transported directly toward the aorta by passing it from the aortic valve. The aortic pressure under HF4 was the same as under HF3 $(113 \mathrm{mmHg})$. The LVAD function not only reduced the LV pressure by mechanical unloading, but also maintained sufficient aortic pressure to support the peripheral and coronary arteries.

Figure $5 \mathrm{f}$ shows the overall ATP consumption rate during a single heart beat in both LVAD and control groups under HF1, HF2, HF3, and HF4 conditions. Generally, a more severely failing ventricle consumed less ATP for myocardial shortening. Furthermore, the LVAD group consumed lower ATP when compared to the control group (Fig. 5f). Specifically, LVAD therapy reduced contractile ATP consumption by $10 \%$ in $\mathrm{HF} 1,8 \%$ in $\mathrm{HF} 2,17 \%$ in $\mathrm{HF} 3$, and $35 \%$ in HF4. These results demonstrated that LVAD reduced the LV mechanical load and contractile energy consumption, especially under severe HF.

Figure $5 \mathrm{~g}$ presents $\mathrm{LV}$ stroke works for both control and LVAD groups across all HF levels. In a manner similar to the data for the overall ATP consumption rate, the more severely failing ventricle performed less stroke work 
to pump blood. Additionally, the LVAD group performed less stroke work as compared to the control group (Fig. $5 \mathrm{~g}$ ). This was because LVAD assisted the LV to pump blood into the aorta. The LVAD therapy reduced LV stroke work by $60 \%$ in $\mathrm{HF} 1,50 \%$ in $\mathrm{HF} 2,42 \%$ in $\mathrm{HF} 3$, and $77 \%$ in $\mathrm{HF} 4$.

Figure $5 \mathrm{~h}$ and i present LV pressure-volume loops in the control and LVAD groups for all the HF severities. The pressure-volume loops were shifted to the right as HF severity increased, and shifted back to the left following LVAD therapy. The pressure decreased as $\mathrm{Ca}^{2+}$ transient magnitude diminished which makes less contractility. Tables 3 and 4 show end of diastolic volume (EDV), ESV, stroke volume (SV), and ejection fraction (EF) as obtained from the pressure-volume loops for all HF severities for control and LVAD groups, respectively.

Figure 6 shows the transmural distribution of electrical activation time (Fig. 6a), and MAT and EMD (Fig. 6b). It also shows the average time of MAT and EMD throughout the entire ventricles for different HF severity in LVAD and control groups (Fig. 6c). Overall, MATs were prolonged for increasing severity of HF while all the EATs were constant. Therefore, more severe HF resulted in longer EMDs (Fig. 6b). The spatial average of MATs was $159 \mathrm{~ms}$ in HF1, $160 \mathrm{~ms}$ in HF2, $162 \mathrm{~ms}$ in HF3, and $163 \mathrm{~ms}$ in HF4. Therefore, the spatial average of EMDs was 79, 81, 82, and $83 \mathrm{ms,}$ respectively. LVAD therapy reduced MAT at each severity level and thereby also reduced EMD. The LVAD therapy reduced the average MAT by $1 \%$ in $\mathrm{HF} 1,2 \%$ in $\mathrm{HF} 2,3 \%$ in HF3, and $6 \%$ in HF4. Therefore, average EMDs were reduced by $1,2,4$, and $18 \%$, respectively. Results indicated that both MAT and EMD were reduced by the mechanical unloading even under mild HF conditions (HF1) in the control group. Thus, LVAD reduced MAT and EMD in all cases.

\section{Discussion}

This study used a sophisticated computational approach (Fig. 1) to predict the effect of $\mathrm{Ca}^{2+}$ remodeling and LVAD function on the cardiac unloading and electromechanical delay in a three-dimensional space of an image-based failing ventricle. In order to take the HF properties into account, we reduced the electrical conductivities, increased

Table 3 EDV, ESV, SV, and EF of control groups (in mL except for $\mathrm{EF})$

\begin{tabular}{lllll}
\hline Control & EDV & ESV & SV & EF \\
\hline HF1 & 87 & 54 & 33 & $40 \%$ \\
HF2 & 93 & 65 & 28 & $30 \%$ \\
HF3 & 100 & 78 & 22 & $22 \%$ \\
HF4 & 104 & 91 & 13 & $13 \%$ \\
\hline
\end{tabular}

Table 4 EDV, ESV, SV, and EF of LVAD groups (in mL except for $\mathrm{EF})$

\begin{tabular}{lllll}
\hline LVAD & EDV & ESV & SV & EF \\
\hline HF1 & 75 & 51 & 24 & $32 \%$ \\
HF2 & 83 & 63 & 20 & $24 \%$ \\
HF3 & 88 & 71 & 0 & Fully assisted \\
HF4 & 86 & 71 & 0 & Fully assisted \\
\hline
\end{tabular}

the myocardial stiffness, and remodeled the calcium transient. In this study, we mainly vary the $\mathrm{Ca}^{2+}$ transient by scaling its magnitude from 1 to 0.7 to mimic the varying HF severities characterized by systolic dysfunction. Despite other remodeling in HF, Constantino et al. [5] reported that primary reason for the prolonged EMD was deranged $\mathrm{Ca}^{2+}$ handling; hence, we varied only the $\mathrm{Ca}^{2+}$ transient magnitude. For each level of HF, two groups were considered: an LVAD treatment group and a control group. We also quantified how the load prolonged the mechanical contraction in four HF severities by a single-cell simulation. Major variables of electromechanical responses were observed including myocardial tension, ATP consumption, strain, LV pressure, aortic pressure, EF, MAT, and EMD. The following were the main findings of this study:

1. Failure in the cardiac myocyte induced by $\mathrm{Ca}^{2+}$ transient remodeling prolonged MAT and EMD (Figs. 3, 4, and 6);

2. The mechanical load prolonged MAT and EMD (Figs. 3 and 4);

3. The remodeled $\mathrm{Ca}^{2+}$ transient prolonged MAT throughout the ventricles (Fig. 6). It also reduced the contractile tension, which reduced the LV and aortic pressures, ATP consumption rate, and LV stroke work and increased the end-diastolic strain (Fig. 5);

4. LVAD shortened MAT (hence, EMD as well) throughout the ventricles (Fig. 6) and reduced the contractile tension, which reduced the LV pressure but increased the aortic pressure, ATP consumption rate, LV stroke work, and end-diastolic strain (Fig. 5).

Several clinically significant responses such as ATP consumption rate, tension, equivalent cell length, LV and aortic pressures, LV pressure-volume diagram, and LV stroke work were examined. We found that these responses worsened with HF severity. However, as we hypothesized here, improvements in ventricular pumping performance due to LVAD were even higher under more severe HF conditions. The results indicate that even though LVAD improved the heart pumping, the peak of LV pressure in the LVAD group was slightly higher compared to that of the control group in the case of HF1, which represented a mild HF condition. 
a
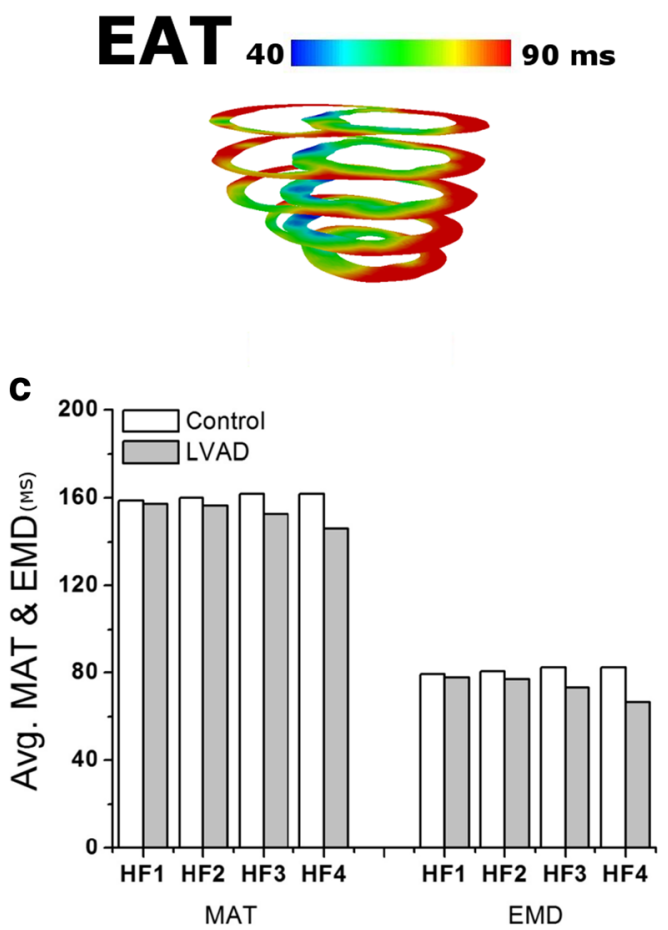

Fig. 6 a 3D distribution of electrical activation time taken when the membrane potential of each node is above 0 ; $\mathbf{b}$ mechanical activation time based on $10 \%$ strain shortening and electromechanical delay,

A computational study by $\mathrm{Yu}$ et al. showed that CRT reduced the ATP consumption by up to $20 \%$ by pacing in the anterior between the apex and base in left bundle branch block (LBBB) condition [11]. This study showed that LVAD reduced the ATP consumption by up to $35 \%$ in severe HF. Therefore, LVAD treatment in combination with CRT can be considered for patients who suffer severe DHF in order to reduce the ventricular energetic load.

EMD as well as MAT increased as the HF severity increased. An increase in the HF severity lowered the $\mathrm{Ca}^{2+}$ concentration in the cardiac muscle, resulting in longer MAT and EMD. LVAD reduced MAT and EMD via mechanical unloading in all HF cases (Fig. 6). LVAD also reduced the ventricular pressure, volume, tension, and ATP consumption (Fig. 5). In contrast to the longer EMD under more severe HF conditions, LVAD performed better by providing a larger reduction in MAT and EMD due to the higher reduction in mechanical unloading.

The results indicated that the differences in the cardiac responses between LVAD and control groups were not significant under mild HF conditions. Thus, the heart does not necessarily need LVAD under normal conditions. By contrast, under the most severe HF, the LV and aorta pressures, energy consumption, stroke work, tension, strain activation, MAT, and EMD showed significant improvements under LVAD treatment.

\section{MAT \\ 80 \\ $200 \mathrm{~ms}$}
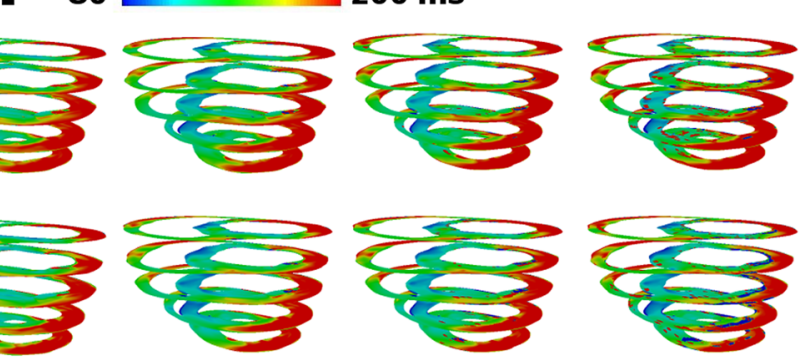

EMD

30

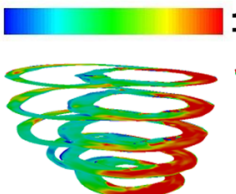

$115 \mathrm{~ms}$
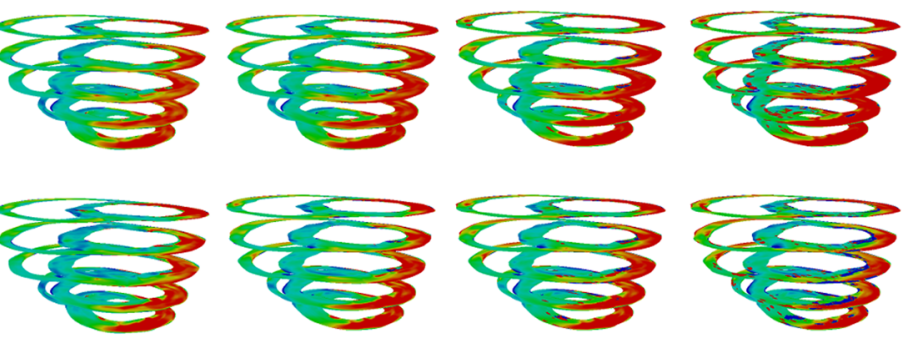

HF1

HF2

HF3

HF4

which is the interval time of EAT and MAT; (c) and average time of MAT and EMD in HF1, HF2, HF3, and HF4

There are some limitations of this study. In our electromechanical model of a failing ventricle, we did not consider the coronary circulation to simplify the computation. We also did not consider the long-term recovery effect of LVAD to the ventricle, whereas previous studies have found that LVAD assists the HF recovery [2, 3, 14, 17]. It may be necessary to consider additional HF properties to quantify the LVAD contribution to EMD under various HF conditions in the future. For instance, a subset of HF in which the heart exhibits dyssynchrony contractions due to the LBBB could be a useful variation. In addition, the integration of autonomic nervous system model for peripheral vascular resistance dynamic would also have a significant impact to examine the correlation between HF and the peripheral vascular system. Nevertheless, the findings of this study present a first step in quantifying the effect of mechanical unloading on cardiac EMD.

\section{Conclusions}

In conclusion, LVAD shortens EMD by mechanical unloading in mild HF, and its performance increases with the severity of the HF. This computational study validated the hypothesis that the LVAD can shorten EMD by mechanical unloading in the ventricle. 
Funding information This research was partially supported by the MSIP, Korea, under the CITRC support program (IITP-2017-2014-000639) supervised by the IITP and NRF (2016R1D1A1B01014405 and 2016M3C1A6936607).

Open Access This article is distributed under the terms of the Creative Commons Attribution 4.0 International License (http:// creativecommons.org/licenses/by/4.0/), which permits unrestricted use, distribution, and reproduction in any medium, provided you give appropriate credit to the original author(s) and the source, provide a link to the Creative Commons license, and indicate if changes were made.

\section{References}

1. About heart failure. http://www.heart.org/HEARTORG/Conditions/ HeartFailure/AboutHeartFailure/About-Heart-Failure_UCM_0020 44_Article.jsp. Accessed date: 20 September 2015

2. Altemose GT, Gritsus V, Jeevanandam V, Goldman B, Margulies KB (1997) Altered myocardial phenotype after mechanical support in human beings with advanced cardiomyopathy. The Journal of Heart and Lung Transplantation: the Official Publication of the International Society for Heart Transplantation 16(7):765-773

3. Barbone A, Oz MC, Burkhoff D, Holmes JW (2001) Normalized diastolic properties after left ventricular assist result from reverse remodeling of chamber geometry. Circulation 104(suppl 1):I-229

4. Berenfeld O, Jalife J (1998) Purkinje-muscle reentry as a mechanism of polymorphic ventricular arrhythmias in a 3-dimensional model of the ventricles. Circ Res 82(10):1063-1077

5. Constantino J, Hu Y, Lardo AC, Trayanova NA (2013) Mechanistic insight into prolonged electromechanical delay in dyssynchronous heart failure: a computational study. Am J Physiol Heart Circ Physiol 305(8):H1265-H1273

6. Constantino J, Hu Y, Trayanova NA (2012) A computational approach to understanding the cardiac electromechanical activation sequence in the normal and failing heart, with translation to the clinical practice of crt. Prog Biophys Mol Biol 110(2):372-379

7. Durrer D, Van Dam RT, Freud G, Janse M, Meijler F, Arzbaecher $\mathrm{R}$ (1970) Total excitation of the isolated human heart. Circulation 41(6):899-912

8. Gurev V, Constantino J, Rice J, Trayanova N (2010) Distribution of electromechanical delay in the heart: insights from a threedimensional electromechanical model. Biophys J 99(3):745-754

9. Gurev V, Lee T, Constantino J, Arevalo H, Trayanova NA (2011) Models of cardiac electromechanics based on individual hearts imaging data. Biomech Model Mechanobiol 10(3):295-306

10. Holzapfel GA, Ogden RW (2009) Constitutive modelling of passive myocardium: a structurally based framework for material characterization. Philosophical Transactions of the Royal Society of London A: Mathematical, Physical and Engineering Sciences 367(1902):3445-3475

11. Hu Y, Gurev V, Constantino J, Trayanova N (2014) Optimizing cardiac resynchronization therapy to minimize atp consumption heterogeneity throughout the left ventricle: a simulation analysis using a canine heart failure model. Heart Rhythm 11(6):1063-1069

12. Jongsma HJ, Wilders R (2000) Gap junctions in cardiovascular disease. Circ Res 86(12):1193-1197

13. Kerckhoffs RC, Neal ML, Gu Q, Bassingthwaighte JB, Omens $\mathrm{JH}$, McCulloch AD (2007) Coupling of a 3d finite element model of cardiac ventricular mechanics to lumped systems models of the systemic and pulmonic circulation. Ann Biomed Eng 35(1):1-18
14. Levin HR, Oz MC, Chen JM, Packer M, Rose EA, Burkhoff D (1995) Reversal of chronic ventricular dilation in patients with end-stage cardiomyopathy by prolonged mechanical unloading. Circulation 91(11):2717-2720

15. Lim KM, Constantino J, Gurev V, Zhu R, Shim EB, Trayanova NA (2012) Comparison of the effects of continuous and pulsatile left ventricular-assist devices on ventricular unloading using a cardiac electromechanics model. J Physiol Sci 62(1):11-19

16. Lloyd-Jones D, Adams R, Carnethon M, De Simone G, Ferguson TB, Flegal K, Ford E, Furie K, Go A, Greenlund K et al (2009) Heart disease and stroke statistics-2009 update a report from the american heart association statistics committee and stroke statistics subcommittee. Circulation 119(3):e21-e181

17. Nakatani S, McCarthy PM, Kottke-Marchant K, Harasaki H, James KB, Savage RM, Thomas JD (1996) Left ventricular echocardiographic and histologic changes: impact of chronic unloading by an implantable ventricular assist device. J Am Coll Cardiol 27(4):894-901

18. Rice JJ, Wang F, Bers DM, De Tombe PP (2008) Approximate model of cooperative activation and crossbridge cycling in cardiac muscle using ordinary differential equations. Biophys $\mathrm{J}$ 95(5):2368-2390

19. Russell K, Smiseth OA, Gjesdal O, Qvigstad E, Norseng PA, Sjaastad I, Opdahl A, Skulstad H, Edvardsen T, Remme EW (2011) Mechanism of prolonged electromechanical delay in late activated myocardium during left bundle branch block. Am J Physiol Heart Circ Physiol 301(6):H2334-H2343

20. Taggart P, Sutton PM, Opthof T, Coronel R, Trimlett R, Pugsley W, Kallis P (2000) Inhomogeneous transmural conduction during early ischaemia in patients with coronary artery disease. $\mathrm{J}$ Mol Cell Cardiol 32(4):621-630

21. Ten Tusscher K, Noble D, Noble P, Panfilov A (2004) A model for human ventricular tissue. Am J Physiol Heart Circ Physiol 286(4):H1573-H1589

22. Vadakkumpadan F, Arevalo H, Prassl AJ, Chen J, Kickinger F, Kohl P, Plank G, Trayanova N (2010) Image-based models of cardiac structure in health and disease. Wiley Interdiscip Rev Syst Biol Med 2(4):489-506

23. What is a ventricular assist device? http://www.nhlbi.nih.gov/ health/health-topics/topics/hvd. Accessed date: 31 March 2015

24. Wu Y, Bell SP, Trombitas K, Witt CC, Labeit S, LeWinter MM, Granzier H (2002) Changes in titin isoform expression in pacinginduced cardiac failure give rise to increased passive muscle stiffness. Circulation 106(11):1384-1389

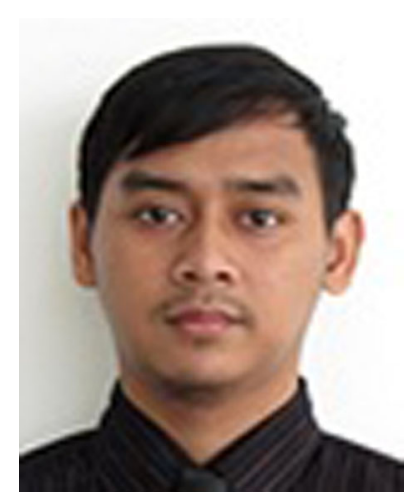

Aulia Khamas Heikhmakhtiar is a Ph.D. student at Kumoh National Institute of Technology, Republic of Korea. His research interest is the cardiovascular system with computational methods. 

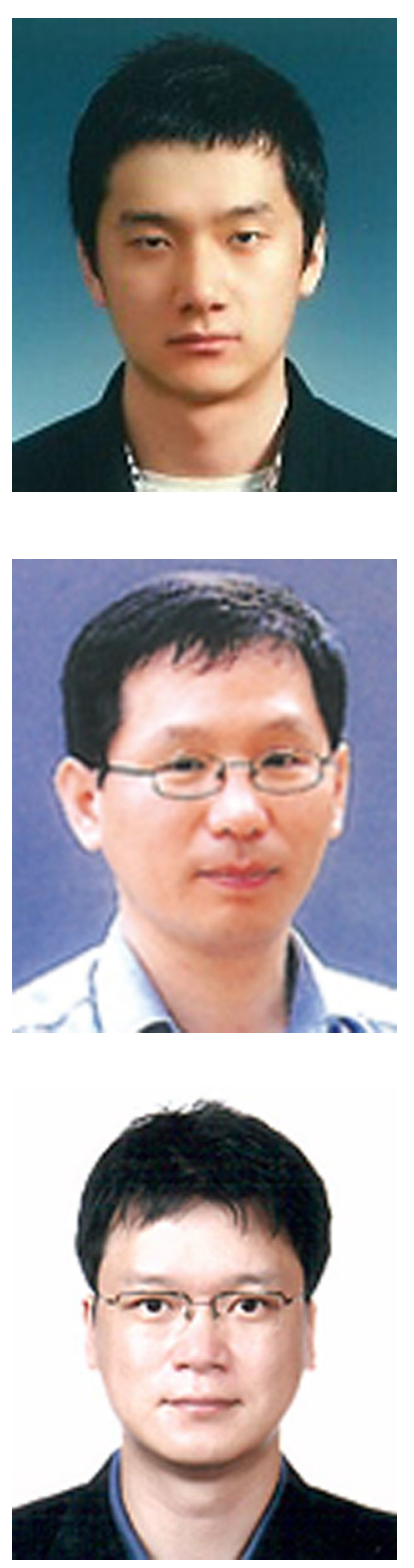

Ah Jin Ryu is a Ph.D. candidate in the Department of Mechanical \& Biomedical Engineering at Kangwon National University, Republic of Korea. His research interest is numerical method of electrophysiology especially in cardiovascular system.

Eun Bo Shim is a Professor of Mechanical \& Biomedical Engineering at the Kangwon National University, Republic of Korea and Director of the National Research Laboratory (NRL) on Biosystems Engineering funded by the Korean Government.

Kwang-Soup Song is an Assistant Professor in Department of Medical IT Convergence Engineering at Kumoh National Institute of Technology, Republic of Korea, with research interests in the biosensors.

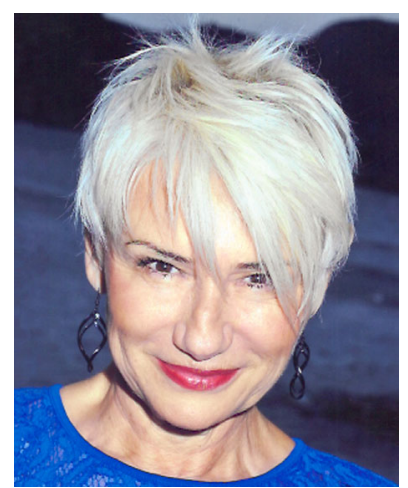

Natalia A. Trayanova is a Professor in the Department of Biomedical Engineering and the Institute for Computational Medicine at Johns Hopkins University, Baltimore, and the inaugural Murray B. Sachs Chair in Biomedical Engineering.

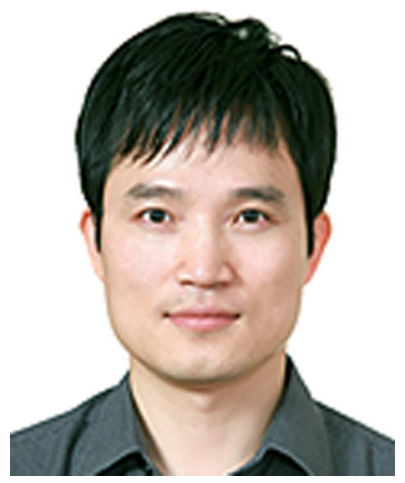

Ki Moo Lim is an Associate Professor of Medical IT Convergence Engineering in Kumoh National Institute of Technology, Republic of Korea. He was a post-doctoral researcher in Kangwon National University, Republic of Korea from 2008 to 2010 and in Johns Hopkins University, USA from 2010 to 2011. 